\title{
Report on the 2010 In Vitro Alternatives Forum
}

Old Town Alexandrian, VA, USA, 18-19 October 2010

The Institute for In Vitro Sciences (IIVS), Inc. presented its third In Vitro Alternatives Forum on 18-19 October 2010 in Old Town Alexandria, VA. The successful event was attended by more than 90 scientists from industry, academia, regulatory agencies, and animal protection organizations. The Forum, which has become the US national meeting on alternatives to animal testing, provided the opportunity for extended discussions of the important in vitro and in silico technology issues facing toxicologists today. The organizing committee - Dan Bagley (Colgate-Palmolive), Rodger Curren (IIVS), Frank Gerberick (P\&G), Thomas Hartung (CAAT, JHU), Robert Landsiedel (BASF), and Ann de Smedt (J\&J) - designed a program covering four main topics: new regulatory chemical testing issues, advances in assessing skin sensitizers, the use of three-dimensional tissue models, and several new and innovative commercially available technologies.

The Forum began by addressing a pervasive new activity that has tremendous international impact - the extraordinary expansion of hazard assessments for new and existing chemicals. The push to conduct more extensive testing has already surfaced in Europe's REACH program and will likely extend to the US via revision of the Toxic Substances Control Act (TSCA). The necessary integration of in vitro and in silico information into these reforms was discussed by a cross-section of interested constituencies. This first session was opened by James Jones, Deputy Assistant Administrator for Chemical Safety and Pollution Prevention, U.S. Environmental Protection Agency (EPA), who discussed details of the potential TSCA revisions and how the EPA is developing ways to utilize non-animal approaches to obtain information that can be used in their decision making process. His presentation was followed by extended comments from three stakeholders (Sara Amundsen, Humane Society Legislative Fund; Richard Becker, American Chemistry Council, and Douglas Fratz, Consumer Specialty Products Association) addressing how their constituencies viewed the potential new legislation.
Continuing with the theme of how to efficiently investigate the potential toxicity of the thousands of chemicals that will be assessed in these new testing programs, Thomas Hartung addressed how experiences from the validation of new non-animal methods over the last decade can be applied as we try to bring the tools of $21^{\text {st }}$ century toxicology to bear on the problem. Robert Kavlock then demonstrated how the EPA's ToxCast program is developing high throughput cellbased methods and the necessary data analysis software that can, hopefully, provide EPA staff with much of the required chemical toxicity information without the use of whole animals. Chihae Yang then described advances in another important tool for chemical toxicity evaluation - computational methods. The final three presentations of the first day offered an overview of EPA's activities with animal alternatives (Tina Levine), a specific EPA program to allow in vitro ocular data to be used for the registration of anti-microbial cleaning products (Rodger Curren), and the informatics and computational safety programs being developed and used by the FDA's Center for Drugs (R. Daniel Benz). It was clear from the first day's presentations that US regulatory agencies are serious about incorporating non-animal test methods, be they cell-based or computational, into the toolbox of approaches that will be used in the safety evaluation of new drugs, chemicals, and products.

Following the first day's formal presentations, Erin Hill (IIVS) and Kristie Sullivan (PCRM) chose the occasion of the 2010 In Vitro Alternatives Forum to make an exciting announcement about the formation of a new scientific society - the American Society for Cellular and Computational Toxicology (ASCCT). One impetus for forming this organization was the existence of several European societies focused on emerging non-animal testing methods and the lack of a single similar society in the US. The ASCCT will provide an organized forum for discussion of cellular and computational toxicology approaches to the safety of chemicals and prod- 
ucts, especially as replacements for animal-based toxicology methods. Through its meetings and activities, the Society will facilitate the development, acceptance, and routine use of cellular and computational methods through open dialogue between industry, academic, advocacy, and regulatory scientists. The Society especially wants to include regulatory scientists in its ranks, as well as to encourage the participation of young scientists who can begin their careers using these important new methods. To support the ASCCT during its first year, a Board of Directors has been appointed. It consists of Mel Anderson, Rodger Curren, Thomas Hartung, Erin Hill, Chad Sandusky, Kristie Sullivan, and Chihae Yang. Information about the society, including how to become a member, can be found by visiting www.ascctox.org or e-mailing info@ascctox.org.

The second day began with a comprehensive look at the remarkable advances being made in the area of in vitro methods to identify skin sensitizers. With the 2013 deadline for the banning of animal tests for skin sensitization imposed by the EU's Seventh Amendment to the Cosmetics Directive approaching, optimism is building that a combination of tests may be available to address a number of steps in the cutaneous immune response. Moderator Robert Landsiedel started the session by reviewing the chain of events leading to skin sensitization and then introduced Anthony Gaspari, Frank Gerberick, David Basketter, Pierre Aeby, Hiroshi Sakaguchi, and William Stokes. The speakers reported on translating human allergic contact dermatitis processes to in vitro systems, peptide reactivity assays, the progress of the ECVAM sensitization prevalidation studies, the Colipa in vitro research program, progress with the h-CLAT assay in Japan, and new LLNA methods.

The Forum then shifted its attention to the various ways that three-dimensional tissue models and ex vivo systems currently are being used for hazard identification and risk assessment within industry. Ann De Smedt, Rodger Curren, James Jester, Marilyn Aardema, Erwin van Vliet, and Marianna Gaça discussed integrating in vitro models into risk assessment, the role of 3D tissue models in $21^{\text {st }}$ century toxicology, histopathology approaches for eye irritation, 3D models in genotoxicity testing, 3D brain cell cultures, and in vitro approaches for inhalation toxicology.
The Forum's final session explored a few of the new commercially available technologies designed to assist toxicologists in evaluating the safety of their chemicals and products. Martin Yarmush of Hurel Corporation presented a microfluidic system for in vitro testing using human cells and tissues, Elizabeth Donley of Stemina Biomarker Discovery described combining stem cells with metabolomics to predict developmental toxicity, and Jayashree Srinivasan of Accelrys Contract Research outlined how their QSTR systems can help predict compound toxicity.

In addition to the invited lectures, conference attendees contributed more than 30 posters describing their findings in multiple areas of in vitro toxicology. Several viewing times were available for the authors to explain their research to fellow scientists.

An additional highlight of the 2010 In Vitro Alternatives Forum was the presentation of the Alternatives Research and Development Fund (ARDF) William and Eleanor Cave Award to the well known and respected journal ATLA (Alternatives to Laboratory Animals). Michael Balls, long-time editor of ATLA, traveled on short notice from the UK to accept the Award presented by Sue Leary, President of ARDF. Sue praised ATLA for being a beacon of hope for non-animal testing through years when progress seemed very slow and significant change appeared unattainable.

The 2010 In Vitro Alternatives Forum covered many of today's most significant topics in non-animal toxicology. Its success lay in the fact that industry scientists, the regulatory community, academics, animal protection groups, and test method suppliers came together to express their individual points of view and to listen respectfully to the views and concerns of other stakeholders.

We anticipate that organization of the next Forum will be carried out by the newly founded ASCCT. The society's diverse membership will provide the collaboration and necessary expertise to broaden and expand the Forum series. IIVS would like to warmly thank this year's speakers, supporters, and attendees who helped to make 2010 Forum a memorable event.

Rodger Curren and Erin Hill Institute for In Vitro Sciences, Inc. 30 W. Watkins Mill Rd., Ste. 100

Gaithersburg, MD 20878, USA 